\title{
« Races guerrières » et masculinité en contexte colonial. Approche historiographique
}

Warrior Races and masculinity in the colonies. A historiographic essay

\section{Vincent Joly}

\section{(2) OpenEdition}

12 Journals

\section{Édition électronique}

URL : https://journals.openedition.org/clio/10054

DOI : $10.4000 /$ clio. 10054

ISSN : 1777-5299

Éditeur

Belin

\section{Édition imprimée}

Date de publication : 1 mai 2011

Pagination : 139-156

ISBN : 978-2-8107-0157-5

ISSN : $1252-7017$

Référence électronique

Vincent Joly, « «Races guerrières » et masculinité en contexte colonial. Approche historiographique », Clio. Femmes, Genre, Histoire [En ligne], 33 | 2011, mis en ligne le 01 mai 2013, consulté le 27 avril 2022. URL : http://journals.openedition.org/clio/10054 ; DOI : https://doi.org/10.4000/clio.10054 


\title{
« Races guerrières » et masculinité en contexte colonial. Approche historiographique
}

\author{
Vincent JOLY
}

Comme l'observe John Mackenzie : "Imperialism seemed to be a very highly gendered phenomenon. Words like "manly", "effeminate", each of them normatively loaded, were seldom far from the lips of imperial rulers $»^{1}$. Cependant, la masculinité est longtemps restée un thème délaissé, les historiens se concentrant plutôt sur l'histoire des femmes dans le monde colonial $^{2}$. Cette focalisation s'explique facilement tant le caractère masculin apparaît comme une évidence du côté du colonisateur. Les Empires sont toujours présentés comme des entreprises masculines illustrées par des hommes blancs en uniforme ${ }^{3}$.

En face, du fait de son statut même de colonisé et donc de vaincu, l'Africain, l'Arabe ou l'Asiatique apparaît comme faible, ayant perdu sa virilité. En bref, il est féminisé. Dès lors, le colonisateur est, selon Mrinalini Sinha, "hyper masculinisé» car il est non seulement le vainqueur, mais il est aussi celui qui définit le genre. Toutefois, cette masculinité n'est pas la transposition d'un simple modèle qui serait construit en métropole et exporté en l'état outre-mer. L'historienne indienne montre que les masculinités européennes et indigènes se construisent sans jamais se figer et qu'elles doivent être analysées dans le cadre des rapports de domination ${ }^{4}$. Elles évoluent au fil du temps avec les changements qui affectent les relations entre colonisateurs et colonisés et demeurent de ce fait des notions

\footnotetext{
Mackenzie 1998 : préface.

Sinha 1999: 446.

Levine 2004 : 1-13.

4 Sinha 1995.
} 
mouvantes, tout comme celle de «races guerrières » dont Heather Streets démontre qu'elle ne peut se comprendre que comme un élément d'une politique coloniale ${ }^{5}$. Les pages qui suivent visent à faire le point à l'aide de quelques travaux récents, en soulignant l'ambiguiité de la notion de «races guerrières » plus particulièrement dans le cas des Empires coloniaux britannique et français.

En 1910, le lieutenant-colonel Charles Mangin publie un livreplaidoyer appelant à la création d'une «force noire $»^{6}$. C'est le fruit d'une enquête de plusieurs mois qu'il a menée officiellement à la demande des autorités locales en Afrique occidentale française ${ }^{7}$. Il y développe deux arguments majeurs. Le premier est: «le fléau de la dépopulation de la France » qu'il impute à la diffusion du bien-être et «de l'ensemble des idées démocratiques $»^{8}$. De ce fait, la participation des Africains à la défense de la France qui est légitime sur le plan moral au nom de la «mission civilisatrice», est une nécessité qu'impose la démographie. Il ne s'agit pas simplement de rétablir l'équilibre démographique avec l'Allemagne, mais aussi de bénéficier d'un atout qualitatif. En effet, et c'est son second argument, "les races nègres ", explique-t-il, "sont conservées dans le même milieu de luttes continuelles qui a renforcé leurs qualités guerrières $»^{9}$. Elles peuvent donc fournir les hommes dont on a besoin a fortiori dans le cadre de la bataille décisive qui doit mener à la victoire car :

C'est surtout dans le choc final que se déploie (sic) la race et sa colère sanguine longuement accumulée qu'aucune dépression nerveuse n’a entamée ${ }^{10}$.

Dans un pays qui a fait du service militaire un élément de régénération nationale et un marqueur de l'identité virile, le sort de la nation repose au moins partiellement sur des colonisés que la « hiérarchie des races » de l'époque juge fondamentalement inférieurs. Le recours à la «force noire » et aux « races guerrières »d'Afrique

\footnotetext{
5 Streets 2004.

6 Mangin 1910.

7 Michel $1974: 83-90$.

8 Mangin $1910: 3$.

9 Mangin $1910: 266$.

10 Mangin $1910: 257$.
} 
peut ainsi témoigner indirectement de la crise de la masculinité que connaît le pays depuis la défaite de 1870. Cette crise est imputée à la « dégénérescence de la race » amollie par la paix, le confort et la ville, mais aussi à l'émergence de cette «femme nouvelle» qui, dans les dernières décennies du XIXe siècle, rejette les valeurs bourgeoises, la maternité et remet en question la domination masculine ${ }^{11}$. Cette crise persiste au-delà de la Grande Guerre. Elle s'aggrave même lorsque s'y ajoutent les séquelles du bain de sang subi entre 1914 et 1918. L'appel aux colonisés apparaît donc comme une nécessité plus pressante encore en 1939. Le 14 juillet de cette année-là, le grandiose défilé militaire qui est organisé à Paris met en vedette les troupes de l'Empire et plus particulièrement les tirailleurs sénégalais que $L a$ Dépêche de Toulouse compare à «de magnifiques statues de bronze» et Paris-Soir aux «noires cariatides de la force française ». Ainsi, à la veille des deux guerres mondiales, à un moment où la France est en proie au doute et où l'idéal masculin doit être mobilisé devant la menace qui pèse sur la nation, la figure du soldat des colonies s'impose comme une image masculine à la fois rassurante et protectrice $^{12}$.

\section{Généalogie de la notion}

\section{de « race guerrière " en contexte colonial}

C'est en Inde que les Britanniques, les premiers, théorisent la notion de «race guerrière». Il existerait des groupes d'hommes biologiquement et culturellement prédisposés aux arts de la guerre. Il y a là un écho du discours alors en cours d'élaboration sur le « racisme scientifique », mais il n'y a pas que cela. En effet, comme le souligne Heather Streets, il ne s'agit pas à l'origine d'une question de couleur car le premier modèle mis en avant dans la deuxième moitié du XVIII siècle est celui du Highlander écossais. Ces hommes, venant d'une rude région vouée à l'élevage, étaient habitués à défendre leurs troupeaux contre leurs voisins et auraient de ce fait développé des aptitudes particulières au combat ${ }^{13}$. Par extension, l'idéologie

\footnotetext{
11 Rauch $2001: 9$; Rearick $1985: 146$.

12 Ageron 1982: 20 ; Surkis $2007: 16$.

13 Streets 2004.
} 
coloniale en France comme en Grande-Bretagne reprend l'idée que les peuples de pasteurs et de montagnards sont intrinsèquement plus « guerriers » que les autres.

Cette notion ne s'épanouit pas en Inde avant la deuxième moitié du XIX siècle. L'East India Company (EIC) qui contrôle le Bengale depuis 1757, avait recruté localement des soldats, les Cipayes, comme les Français l'avaient fait avant elle, sans distinction d'origine sociale ou géographique. À partir de 1800, l'EIC commence à se concentrer sur les castes les plus hautes, Brahmanes et Rajputs, mettant en avant leur grande taille et leur peau plus claire sans pour autant les présenter comme des hommes plus adaptés que d'autres au service militaire ${ }^{14}$. Les piètres performances de l'armée du Bengale au cours de la première guerre de Birmanie (1824-1826), et surtout lors de la mutinerie de Barrackpore en 1826, ont semé le doute parmi les responsables militaires britanniques sur les qualités, le loyalisme et la confiance qu'ils pouvaient accorder à ces hommes. Il s'agit d'une première étape qui conduit les Britanniques à abandonner progressivement un modèle reposant sur les stéréotypes de caste au profit d'une armée fondée sur des stéréotypes raciaux. Désormais, les paysans de l'Oudh et des régions voisines du Bihâr apparaissent comme des hommes robustes, simples et bien plus fiables que les Bengalis, jugés malhonnêtes, faux et donc efféminés ${ }^{15}$.

La révolte des Cipayes en 1857 bouleverse les relations angloindiennes et remet en question les bases de l'organisation militaire. Dans le cadre de la répression, les Britanniques se sont tournés vers les populations qui étaient demeurées loyales. Le Punjab, conquis en 1848, le Népal, soumis en 1816, fournissent Sikhs et Gurkhas qui constituent la base de l'armée des Indes jusqu'à la Deuxième Guerre mondiale et deviennent les archétypes des «races guerrières ». Le soulèvement a convaincu les responsables à Londres que les Indiens étaient dans l'ensemble imperméables à la modernité et que la domination devait s'appuyer sur leurs «traditions orientales » plutôt que de chercher à les transformer ${ }^{16}$. En Angleterre même, les médias

\footnotetext{
14 Metcalf $1995: 125$.

15 Peers $1991: 546$.

16 Metcalf $1995: 28$ sq.
} 
insistent sur les massacres et les viols dont ont été victimes les femmes blanches. Ces crimes deviennent des éléments centraux dans les relations anglo-indiennes et contribuent à les définir non seulement en termes raciaux, mais aussi en termes de genre. Selon l'image de la femme qui domine dans l'Angleterre victorienne, les coupables ne peuvent être des hommes mais sont des barbares conduits par leurs passions et par leurs sens, c'est-à-dire le contraire d'êtres civilisés. Comme le montre Philippa Levine, le traitement des femmes dans une société est alors considéré comme un témoignage de son degré de civilisation. Plus une société est avancée et plus elle se doit de protéger les femmes ${ }^{17}$. Dans ces conditions, la vengeance et les brutalités qui l'accompagnent deviennent légitimes car ce sont des lâches qui ont violé ces femmes. Les soldats britanniques, sikhs et gurkhas, sont alors parés de toutes les vertus chevaleresques et incarnent ainsi la véritable masculinité. Cela signifie qu'ils sont capables de contrôler leur sexualité et de diriger leur énergie vers le travail, la guerre ou encore le sport ${ }^{18}$.

En 1858, une commission est chargée de réformer l'organisation militaire de l'Armée des Indes désormais prise en charge par le gouvernement après la dissolution de l'EIC. Ses conclusions suggèrent de reconstituer l'armée indienne en amalgamant au niveau régimentaire différents groupes ethniques de manière à éviter la prépondérance de l'un d'entre eux. Seuls les Gurkhas échappent à cette règle, et cette exception annonce l'application généralisée de la théorie des " races guerrières » qui intervient à la fin du siècle ${ }^{19}$. Lors du soulèvement des Cipayes, les militaires britanniques avaient estimé que Sikhs et Gurkhas s'étaient ralliés à eux parce qu'ils avaient compris le sens des mots honneur et devoir qui fondent une véritable éthique militaire. Dans les années 1860, ils avaient dégagé un certain nombre de critères permettant de sélectionner les « races guerrières ». Le premier est l'origine des peuples. Les plus aptes seraient ainsi les descendants des Aryens qui auraient conquis l'Inde grâce à leurs compétences militaires supérieures. Cette idée qui supposait une

17 Levine $2004: 1-13$.

18 Streets $2004: 43$.

19 Rand $2006: 7$. 
« égalité raciale » avec les Européens, ne correspondait pas du tout au discours dominant sur l'infériorité et l'altérité des Indiens et, surtout, se révélait peu compatible avec la domination coloniale ${ }^{20}$. Sans surprise, elle disparaît dans le dernier quart du XIX ${ }^{\mathrm{e}}$ siècle. Le second critère est géographique et associe le sol, le climat, le genre de vie. Les populations montagnardes seraient plus aptes à développer leurs qualités martiales que celles vivant en plaine, en zone humide ou encore en ville. En fait, le critère le plus important est le loyalisme, ce qui explique en définitive le flou de la théorie des "races guerrières » et sa très grande flexibilité. C'est surtout un outil de domination politique qui doit pouvoir s'adapter à toutes sortes de circonstances ${ }^{21}$. Le loyalisme est présenté comme éminemment masculin et permet de conférer des traits de caractère féminins à tous ceux qui ne sont pas entièrement acquis aux intérêts du colonisateur, ainsi des intellectuels indiens nationalistes qui deviennent des babus efféminés 22 . Il s'agit donc bien de l'invention d'une race qui prend place dans une vaste entreprise de classification des populations jamais définitivement close.

\section{Le point de vue des colonisés}

Au-delà de cette invention se pose le problème de l'adhésion des colonisés à ce qu'elle signifie et à ce qu'elle implique dans la définition de leur propre masculinité. On peut noter avec David E. Omissi au sujet de l'armée indienne après 1857 que la religion sikhe avait été militarisée au début du XVIII ${ }^{\text {e }}$ siècle pour lutter contre l'oppression moghole. De plus, certaines castes elles-mêmes se considéraient comme martiales. Ainsi, le dharma d'un Kshatriya lui impose de dominer voire de tuer ses ennemis et les Rajputs (qui sont eux-mêmes un sous-groupe des varnas Kshatriya) se présentent comme des guerriers $^{23}$.

En Afrique de l'Est, la violence est spécifiquement masculine et les conflits sont souvent cause ou conséquence de la capture des

\footnotetext{
20 Leopold 1974 : 598.

21 Streets $2004: 157$ sq.

22 Sinha 1995 : introduction.

23 Omissi $1994: 24$
} 
femmes. En Éthiopie orientale, tuer un homme constitue un rite de passage qui permet à son tour de devenir un homme et d'obtenir une femme ${ }^{24}$. En Afrique de l'Ouest, l'image martiale des Bambara n'est pas uniquement le fruit de l'imagination des Français. C'est bien un État militaire qui s'est installé à Ségou au début du XVIII ${ }^{\mathrm{e}}$ siècle, animé par des combattants professionnels qui ont fait du pillage et de la guerre leurs principaux moyens d'existence ${ }^{25}$. Les colonisateurs ont donc pu penser qu'ils pouvaient réutiliser ce qu'ils prenaient pour des traditions martiales à leur profit. Ce n'est pas toujours faux. Au Kenya, les Nandi se sont engagés parce que l'armée leur offrait la possibilité de conserver un statut de guerrier. Selon John Illife, beaucoup de volontaires expliquaient leur choix en déclarant qu'ils s'étaient engagés "parce qu'ils étaient de hommes » et parce qu'ils voulaient «tester leur virilité $»^{26}$. Ainsi, il n'y a pas forcément «démasculinisation» pour les colonisés lorsqu'ils rejoignent l'armée de leurs vainqueurs. Kandé Kamara interrogé par Joe Lunn, justifie son engagement comme tirailleurs sénégalais non seulement parce qu'il est honteux pour sa famille de ne pas le faire alors que des membres de lignages moins prestigieux l'ont fait mais aussi parce qu'à ses yeux, le service militaire est une preuve de la "masculinité absolue » du guerrier soussou et enfin parce que, dans son esprit, l'expérience de la guerre doit conduire à l'égalité avec les Français ${ }^{27}$. Par ailleurs, la masculinité n'est pas définie par la seule activité guerrière mais aussi par l'âge, l'autorité ou la richesse. De plus, elle est historiquement et culturellement construite ce qui signifie qu'elle demeure une notion plastique, susceptible de s'adapter au contexte colonial. Au Mali, être ancien combattant ne suffit pas si l'on ne peut en même temps affirmer son autorité sur les jeunes et les femmes ${ }^{28}$.

Les « races guerrières» définies par le colonisateur peuvent ainsi adhérer à cette catégorisation même si elle n'a rien à voir avec des traits culturels particuliers ou des traditions précoloniales. Dans l'Inde

\footnotetext{
24 Reid $2007: 208-209$.

25 Roberts $1987: 22$.

26 Illife $2005: 233-234$.

27 Lunn $2005: 713-376$.

28 Lindsay \& Miescher 2003 : introduction.
} 
britannique, selon Cynthia Enloe, il existe un «syndrome Gurkha » qui explique que les groupes ethniques les plus pauvres utilisent le service militaire comme élément définissant leur identité ${ }^{29}$. En s'engageant dans les King's African Rifles, le jeune Kamba cherche non seulement à prouver sa virilité mais aussi à intégrer l'économie coloniale et à bénéficier d'une meilleure considération de la part des autorités britanniques ${ }^{30}$.

\section{Images partagées des masculinités dans les empires anglais et français}

En Inde dans les années 1890, la détection des « races guerrières » fait l'objet d'une classification plus rigoureuse dont témoignent les Recruiting Handbooks qui restent en usage jusqu'à la Deuxième Guerre mondiale. Ces manuels insistent sur «l'indépendance virile» des hommes qui sont déclarés bons pour le service pour mieux les distinguer du caractère efféminé de ceux qui en sont exclus ${ }^{31}$. Les politiques de recrutement abandonnent alors une grande partie de l'Inde pour se concentrer sur le Punjab et le Népal qui fournissent 44\% des effectifs en 1893, mais 75\% en 1914.

Le guerrier, désormais soldat, devient une figure familière dans un environnement marqué par une altérité incompréhensible. Il assimile les normes du comportement masculin occidental comme la pratique des sports dits virils. De même, les officiers britanniques adoptent les sports locaux comme le polo. Ainsi, dans la guerre et le sport, les différences qui fondent l'ordre colonial peuvent s'estomper autour d'une image partagée de la masculinité. Comme le suggère David Cannadine, cette connivence se fonde aussi sur des critères sociaux qui rapprochent l'aristocratie indienne des hauts-fonctionnaires et des officiers britanniques autour de valeurs et d'honneurs communs ${ }^{32}$. La guerre permet un rapprochement plus large dont le fondement est sans doute la même perception de l'idéal masculin. Heather Streets montre comment Highlanders, Sikhs et Gurkhas sont associés

\footnotetext{
29 Enloe 1980.

30 Parsons 1999 : 149 sq.

31 Omissi $1994: 25$.

32 Cannadine $2001: 45$ sq.
} 
comme camarades de combat à partir du soulèvement de 1857, partageant le même esprit de corps à la base de l'homogénéité des régiments. Symboliquement, le commandement suggère à la fin du $\mathrm{XIX}^{\mathrm{e}}$ siècle que toutes ces unités adoptent les mêmes insignes et les mêmes musiques ${ }^{33}$. La guerre est bien le moment privilégié de ce rapprochement, comme en témoigne la culture populaire en GrandeBretagne. Dans le film d'Alexander Korda, The Drum, tourné en 1938, une révolte est brisée grâce à l'alliance entre un capitaine britannique et un prince indien entre lesquels existe une égalité admirative, comme il sied entre combattants chevaleresques. Au-delà de la fiction, la frontière du nord-ouest de l'Inde où les affrontements sont permanents entre Britanniques et Pathans devient le lieu privilégié où, loin des femmes et de la vie amollissante des plaines, on se bat entre hommes. Pour Thomas Metcalf, le Pathan devient un alter ego idéalisé, le demi-barbare caché au fond de la personnalité de l'officier de Sa Majesté ${ }^{34}$.

Dans l'Empire colonial français, si l'on retient la notion de «race guerrière », on ne cherche pas à la théoriser ni même à la systématiser. Cependant, jusqu'à la Première Guerre mondiale, parmi les populations d'Afrique occidentale, les Bambara occupent une place à part au point que leur langue devient celle des régiments de tirailleurs sénégalais. Toutefois, ils ne sont pas les seuls représentants de ces « races guerrières » distinguées par Mangin. En 1918, la Notice sur les Sénégalais et leur emploi an combat leur adjoint les Wolof, les Serer, les Mossi et les «Toucouleurs » qui composent les meilleures unités ${ }^{35}$. Dans cette hiérarchie, le facteur biologique joue un rôle important. En 1875, Léon Figuières « explique » que les Africains ont un système nerveux peu sensible qui leur permet de supporter la douleur. Mangin reprend cet argument et l'oppose à l'extrême nervosité des peuples civilisés, proche de comportements féminins. La culture africaine faite de violences permanentes contribue elle aussi à développer leurs aptitudes martiales. Or, celles-ci tendent à décroittre grâce aux

\footnotetext{
33 Streets $2004: 142$.

34 Metcalf $1995: 146$.

35 Lunn $1999: 521$.
} 
«bienfaits» de la colonisation, entrainant, selon les médecins militaires, la disparition progressive des instincts guerriers ${ }^{36}$.

Il n'y a pas en France de discours unique sur les races guerrières et il n'existe pas en français l'équivalent du livre de référence de Sir G. MacMunn, The Martial Races of India, publié en 1933. La guerre a sans doute changé la vision des Africains en dépit de la persistance de vieux stéréotypes au nom desquels on continue d'affirmer que : «À quelques exceptions près, l'indigène africain a le tempérament guerrier $»^{37}$. Pour autant, la description des groupes ethniques n'insiste pas sur la distinction entre les « races guerrières » et celles qui ne le seraient $\operatorname{pas}^{38}$. Dans l'Histoire militaire de l'Afrique occidentale française publiée par la Direction des troupes coloniales à l'occasion de l'Exposition internationale de Vincennes en 1931, l'expression a quasiment disparu et a laissé la place à une simple opposition entre races blanche et noire ${ }^{39}$. En fait, il n'y a désormais plus de place pour distinguer les "guerriers » car l'armée a fait de tous des soldats. Elle constitue pour le colonisé un milieu plus égalitaire que le monde colonial des civils. Pour Robert Delavignette :

L'administration dit encore les indigènes et le public les nègres. Mais l'officier avait toujours dit les Hommes. Non pas les "grands enfants", niaise dénomination que l'on trouve encore dans de récents discours mais les Hommes tout simplement ${ }^{40}$.

\section{La sexualité et les femmes : une color line infranchissable ?}

Ainsi, la rhétorique militaire officielle tend à faire du colonisé un homme à part entière lorsqu'il est sous l'uniforme ou plus exactement lorsqu'il est soldat. Tyler Stovall relève que pendant la Première Guerre mondiale, les ouvriers coloniaux sont féminisés y compris par les femmes au même titre que les "planqués $»^{41}$. Cette égalité entre les soldats n'est qu'apparente pour au moins deux raisons. La première

\footnotetext{
36 Osborne \& Fogarty 2003 : 37.

37 Manuel à l'usage des troupes employées outre-mer 1923 : 187.

38 Michel $1992: 315$.

39 Histoire militaire de l'Afrique occidentale française 1931 : 26-28.

40 Delavignette $1931: 154$.

41 Stovall $1998: 764$, n. 115.
} 
est que si les qualités physiques des Africains sont reconnues voire jugées supérieures à celles des Européens, ces derniers conservent l'intelligence qui justifie leur domination. La seconde tient à la sexualité. Ann Stoler montre combien les catégories de «colonisateur» et de «colonisé » sont littéralement cadenassées par un contrôle sexuel qui définit à la fois l'intérêt politique des Européens et la manière dont ils s'envisagent eux-mêmes par rapport aux Africains ou aux Asiatiques ${ }^{42}$. Ainsi, comme le rappelle avec force Mangin, l'appel aux colonisés se limite au champ de bataille. Depuis le milieu du XIX ${ }^{e}$ siècle, le métissage est devenu un "problème angoissant». Les anthropologues estiment qu'il ne donne que des individus "d'une infériorité évidente » moralement et physiquement. Mais, surtout, leur existence apparaît aussi comme une remise en question de la hiérarchie coloniale ${ }^{43}$.

La sexualité des colonisés demeure une préoccupation permanente d'autant plus que, pendant la guerre en métropole, tirailleurs africains et asiatiques côtoient des Européennes et que celles-ci peuvent succomber à leurs charmes. C'est en tout cas ce que chantent les lecteurs de Crache pas dans l'masque, le journal de la 3e Division Coloniale en 1916 :

Depuis que dans l'Nord de la France et ailleurs

S'sont amenés des contingents étrangers

D’Tonkinois, de Bédouins, d'Malgaches, de Toucouleurs

Dans l'pays les p'tites femmes sont aux anges

Ell's ne peuvent s'empêcher de zyeuter l'pantalon

De ces noirs... ${ }^{4}$

Ce thème est inépuisable et perdure après la guerre dans la littérature populaire. Ainsi, l'héroïne du roman de Pierre Mille et André Demaison, deux auteurs coloniaux, La Femme et l'homme nu, séduit un jeune tirailleur en convalescence à Fréjus :

Les confidences de sa camériste [...] les racontars extraordinaires de la ville habituée depuis trois ans aux noirs des camps de tirailleurs, sont pour elle des indications troublantes, apparemment exagérées [...] Elle

\footnotetext{
42 Stoler 1989.

43 Saada 2007: 27 sq.

44 Liauzu 2002 : 92-93.
} 
brûle de les contrôler. Ses sens qu'elle croyait endormis en souhaitent l'exactitude ${ }^{45}$.

La virilité des Européens est doublement remise en cause. Leurs femmes sont des proies potentielles voire des victimes consentantes et, en plus, les colonisés se présentent comme les sauveurs de la Nation. Il faut donc éviter au maximum les contacts. L'instauration des bordels mobiles de campagne, si possible avec des prostituées nord-africaines, et la création de maisons de tolérance qui leur sont réservées dans les villes de l'arrière, deviennent des nécessités à la fois politiques et sociales ${ }^{46}$. Comme le note Richard Fogarty, la transgression est aussi imputée aux femmes. Les autorités militaires blâment leurs comportements jugés indécents et la censure traque les lettres dans lesquelles les soldats d'outre-mer se vantent de leurs succès féminins ${ }^{47}$. Les infirmières qui servent dans les hôpitaux militaires sont particulièrement visées. Elles sont accusées de " pourrir» les tirailleurs blessés qui en sortent «pleins d'arrogance». Il faut donc les «resénégaliser » selon l'expression d'un fonctionnaire colonial, en les éloignant de toute présence féminine ${ }^{48}$.

Les Empires coloniaux comme l'armée sont des espaces masculins. Dans les casernes, dans les postes de brousse ou les fortins du désert, on vit entre hommes, loin des femmes. Dans le film de Maurice Gleize, Légion d'honneur, présenté en 1937, Charles Vanel qui joue le rôle d'un capitaine méhariste, affirme à son jeune adjoint : «Le bled ça unit, les femmes ça sépare $»^{49}$. Cette promiscuité peut banaliser l'homosexualité qui est pourtant présentée comme le contre-modèle du courage militaire et donc de la masculinité. Or, au point de vue du sexe comme de la morale, l'environnement colonial est perçu comme clairement distinct de la métropole. En bref, ce qui est intolérable en France ou en Grande-Bretagne peut être toléré

\footnotetext{
45 Ruscio $1996: 811$.

46 Joly $2007: 68$ sq.

47 Fogarty $2008: 310$.

48 Mann $2006: 166$.

49 Boulanger 1975 : 96.
} 
outre-mer et les Empires deviennent ainsi des lieux d'expériences sexuelles ${ }^{50}$.

L'image de la virilité n'est alors plus incompatible avec l'homosexualité. S'inspirant sans doute de la Grèce antique, un officier britannique servant dans les Gurkhas, estime que l'homosexualité renforce l'esprit de corps et les comportements martiaux de ces soldats d'élite. Il oppose leur comportement à celui des homosexuels occidentaux ou bengali, définitivement féminisés ${ }^{51}$. Les médecins militaires français en Algérie se penchent très tôt sur cette «déviance». Selon eux, la solitude, l'absence de femmes la favorisent mais aussi le climat et les mœurs locales supposées. En 1845, le docteur Bodichon est convaincu que la chaleur encourage les «dérèglements » de la sexualité parmi lesquels il range la polygamie et l'homosexualité, cette dernière étant dénommée "vice oriental ${ }^{52}$. Les milieux militaires ne perçoivent pas l'homosexualité comme une menace dans la mesure où elle ne remet pas en cause l'efficacité et la cohésion des unités. Elle ne concerne pas seulement les troupes indigènes mais aussi les soldats européens qui servent outre-mer. Ainsi, dans les bataillons d'Afrique, au sein d'une unité disciplinaire en garnison dans le sud de la Tunisie, une enquête menée en 1907 estime aux deux-tiers la proportion d'homosexuels ${ }^{53}$. Certes, les médecins militaires expliquent ce phénomène par le passé criminel de ces hommes souvent, en plus, mentalement dérangés, mais à aucun moment, ils ne remettent en question leurs qualités de combattants. De même, personne ne s'interroge sur la virilité des légionnaires espagnols du Tercio bien que Milan Astray, l'un de ses créateurs, insiste sur leur travestissement parmi leurs formes de récréation collective ${ }^{54}$. En revanche, Douglas Porch estime que dans la Légion étrangère française, l'homosexualité répandue dans les postes du Sud-

\footnotetext{
50 Hyam $2010: 364$.

51 Omissi $1994: 26$.

52 Lorcin $1999:$ 653-679.

53 Aldrich $2003: 60$.

54 Martin-Marquez 2004 : 233.
} 
algérien «crée une atmosphère malsaine, brise les amitiés et donne naissance à des sentiments de méfiance et de dégoût $»^{55}$.

L'homophilie est sensible dans les relations entre officiers européens. L'ancien «blédard » initie le «bleu» à sa nouvelle vie. On retrouve ces relations dans un roman qui connait un grand succès entre les deux guerres en France, La châtelaine du Liban de Pierre Benoit (1924). Le lieutenant Domène en route vers Palmyre, évoquant ses liens avec le capitaine Walter qu'il va rejoindre, se confie à lui-même: «Elle m'attendait cette amitié virile et pure. J'allais pouvoir tout ensemble m'y blottir et m'y laver ${ }^{56}{ }^{5}$. Les témoignages romancés ou non sur les relations homosexuelles entre militaires européens et indigènes sont très rares. La color line semble ici infranchissable mais comme le note Douglas Peers dans le cas de l'armée des Indes, l'institution refusait tout simplement d'en reconnaître l'existence et faisait tout pour étouffer la moindre affaire ${ }^{57}$. Ce silence peut être rompu par la tragédie comme le suicide à Ceylan en 1903 de Sir Hector Macdonald, un héros de la guerre anglo-boer, surpris avec de jeunes Cinghalais. Le scandale vient sans doute moins de cette homosexualité révélée que du fait qu'elle implique des colonisés et qu'elle entame ainsi le prestige du colonisateur ${ }^{58}$.

Pour les autorités coloniales, la notion de «race guerrière » est ainsi davantage un outil politique qu'un critère de recrutement militaire, ce qui explique le flou qui entoure sa définition et les changements quelle connaît tout au long de la période coloniale. L'expérience de la Première Guerre mondiale a, de plus, montré dans le cas des Africains que les soldats recrutés dans les groupes n'ayant pas a priori d'aptitudes militaires, montraient au combat autant de qualités que les autres ${ }^{59}$. Bien que porteuses d'un idéal viril, celui des combattants, les «races guerrières » inventées par le colonisateur ne

\footnotetext{
55 Porch $1994: 370$.

56 Benoit $1986: 274$.

57 Peers $1998: 823-854$.

58 Hyam $2010: 366$.

59 Bayo Adeson $1979:$ 157-165.
} 
peuvent représenter une masculinité idéale. Celle-ci reste définie par le colonisateur et devient aussi un élément du discours de la domination. Comme le disent avec un mélange d'admiration et d'effroi les poilus dépeints par Henri Barbusse dans Le Feu:

L'sont vraiment d'une autre race que nous, avec leur peau en toile de tente, avoue Biquet qui, pourtant n'a pas froid aux yeux. Le repos les embête, tu sais; ils ne vivent que pour le moment où l'officier remet sa montre dans sa poche et dit: "Allez! Partez !" - Au fond, ce sont de vrais soldats. - Nous ne sommes pas des soldats, nous, nous sommes des hommes. ${ }^{60}$

\section{Bibliographie}

AGERON Charles-Robert, 1982, «La perception de la puissance française en 19381939 : le mythe impérial », RFHOM, 254, p. 7-22.

AlDrich Robert, 2003, Colonialism and Homosexuality, Londres, Routledge.

Barbusse Henri, 1994 [1916], Le feu. Journal d'une escouade, in Les grands romans de la guerre 14-18, Paris, Omnibus, p. 11-250.

BAyo Adeson James, 1979, "Ethnicity and Army recruitment in Colonial Plural Society ", Ethnic and Racial Studies, 2, p. 157-165.

Benoit Pierre, 1986 [1924], La Châtelaine du Liban, Paris, Albin Michel.

Boulanger Pierre, 1975, Le cinéma colonial. De l'Atlantide à Lawrence d'Arabie, Paris, Seghers.

Cannadine David, 2001, Ornementalism. How the British saw their Empire, Londres, Penguin Books.

Delavignette Robert, 1931, Afrique Occidentale Française, Paris, Société d'éditions géographiques, maritimes et coloniales.

EnLoe Cynthia, 1980, Ethnic Soldiers: State Security in a Divided Society, Athens, University of Georgia Press.

FOgARTY Richard, 2008, Race and War in France. Colonial Subjects in the French Army, 1914-1918, Baltimore, The Johns Hopkins University Press.

Histoire Militaire de l'Afrique occidentale francaise, Paris, Imprimerie nationale, 1931.

Hyam Ronald, 2010, Understanding the British Empire, Cambridge, Cambridge University Press.

60 Barbusse 1994 [1916] : 41. 
IlliFe John, 2005, Honour in African History, Cambridge, Cambridge University Press. Joly Vincent, 2007, « Sexe, guerres et désir colonial », in François RouQuET, Fabrice Virgili \& Danièle Voldman (dir.), Amours, guerres et sexualité, 1914-1945, Paris, Gallimard, p. 62-69.

LEOPOLD Joan, 1974, "British Application of the Aryan Theory of Race to India », The English Historical Review, 89/352, p. 578-603.

Levine Philippa, 2004, "Why Gender and Empire? », in P. LEvine (ed.), Gender and Empire, Oxford, Oxford University Press, p. 1-13.

LiauZu Claude \& Josette, 2002, Quand on chantait les colonies, Paris, Syllepse.

Lindsay Lisa A. \& Stephan Miescher (eds), 2003, Men and Masculinities in Modern Africa, Portsmouth, Heinemann.

LORCIN Patricia, 1999, "Imperialism, Colonial Identity and Race in Algeria, 18301870: The Role of the French Medical Corps ", Isis, 90/4, p. 653-679.

LunN Joe, 1999, "Les Races Guerrières”: Racial Preconceptions in the French Military about West African Soldiers during the First World War », Journal of Contemporary History, 34/4, p. 517-536.

—, 2005, «Male Identity and Martial Codes of Honour: A Comparison of the War Memoirs of Robert Graves, Ernst Jünger and Kande Kamara », The Journal of Military History, 69, p. 713-736.

MackenzIE John, 1998, "Préface », in Claire Midgley (ed.), Gender and Imperialism, Manchester, Manchester University Press.

MANGIN Charles, 1910, La force noire, Paris, Hachette.

ManN Gregory, 2006, Native Sons. West African Veterans and France in the Twentieth Century, Durham, Duke University Press.

Manuel à l'usage des troupes employées outre-mer, Paris, Lavauzelle, 1923.

Martin-Marquez Susan, 2004, «Performing Masculinity in the Moroccan Theatre: Virility, Sexuality and Spanish Military Culture from the African War to the Civil War », European Review of History - Revue Européenne d'Histoire, 11/2, p. 225-240.

Metcalf Thomas R., 1995, Ideologies of the Raj, Cambridge, Cambridge University Press.

MiCHEL Marc, 1974, «Un mythe: la "force noire" avant 1914 », Relations Internationales, 1, p. 83-90.

—, 1992, «Les peuples et l'histoire de l'Afrique dans les manuels d'instruction militaire entre les deux guerres ", in Colette Dubois, Hubert GerbeAu, Yvan Paillard \& Pierre Soumille (dir.), Histoire d'Outre-Mer. Mélanges offerts à J.L. Miège, Aix-en-Provence, PUP, p. 313-328. 
Midgley Claire (ed.), 1998, Gender and Imperialism, Manchester, Manchester University Press.

Omissi David E., 1994, The Sepoy and the Raj. The Indian Army, 1860-1914, Houndmills, Macmillan.

Osborne Michael A. \& Richard Fogarty, 2003, «Views from the Periphery: Discourses of Race and Place in French Military Medicine ", Hist. Phil. of Life Sciences, 25, p. 363-389.

Parsons Thimoty H., 1999, The African Rank and File. Social Implications of Colonial Military Service in the King's African Rifles, 1902-1964, Portsmouth, Heinemann.

PeErs Douglas, 1991, "The Habitual Nobility of being: British Officers and the social Construction of the Bengal Army in the early Nineteenth Century ", Modern Asian Studies, 25/3, p. 545-569.

—, 1998, «Private off Parades: Regimenting Sexualities in the Nineteenth Century Empire », International History Review, 20, p. 823-54.

PORCH Douglas, 1994, La Légion étrangère, 1831-1962, Paris, Fayard.

RAND Gavin, 2006, « Martial Races and Imperial Subjects: Violence and Governance in colonial India, 1857-1914», European Review of History - Revue Européenne d'Histoire, 13/1, p. 1-20.

RAUCH André, 2001, Crise de l'identité masculine, 1789-1914, Paris, Hachette.

REARICK Charles, 1985, Pleasures of the Belle Époque, New Haven, Yale University Press.

REID Richard, 2007, War in Pre-Colonial Eastern Africa, Oxford, James Currey.

REVENIN Régis (dir.), 2007, Hommes et masculinités de 1789 à nos jours, Paris, Autrement.

RoBerTs Richard L., 1987, Warriors, Merchants and Slaves. The State and the Economy in the Middle Niger V alley (1700-1914), Stanford, Stanford University Press.

Ruscio Alain, 1996, Amours coloniales. Aventures et fantasmes exotiques de Claire Duras à Georges Simenon, Bruxelles, Complexe.

SAADA Emmanuelle, 2007, Les enfants de la colonie. Les métis de l'Empire français entre sujétion et citoyenneté, Paris, La Découverte.

SINHA Mrinalini, 1995, Colonial Masculinity. The "Manly Englishman" and the "Effeminate Bengali" in the late Nineteenth Century, Manchester, Manchester University Press.

SINHA Mrinalini, 1999, "Giving masculinity a History: Some Contributions from the Historiography of Colonial India », Gender and History, 11/3, p. 445-460.

Stoler Ann L., 1989, « Making Empire Respectable: The Politics of Race and Sexual Morality in $20^{\text {th }}$ Century Colonial Cultures ", American Ethnologist, 4/3, p. 634-660. 
StOvall Tyler, 1998, «The Color Line behind the lines: Racial Violences in France during the Great War », American Historical Review, 103/3, p. 737-769.

STREeTs Heather, 2004, Martial Races. The Military, Race and Masculinity in British Imperial Culture, 1857-1914, Manchester, Manchester University Press.

SuRKIS Judith, 2007, " Histoire des hommes et des masculinités : passé et avenir », in Régis REVEnIN (dir.), Hommes et masculinités de 1789 à nos jours, Paris, Autrement, p. 13-20. 